\title{
The research of synchronization algorithm based on Zigbee
}

\author{
Ping Wang ${ }^{1, a}$, ZhangBin Chen ${ }^{2, b}$ \\ ${ }^{1}$ Fuzhou College of foreign studies and trade, Fuzhou, China, 350202 \\ ${ }^{2}$ Fuzhou College of foreign studies and trade, Fuzhou, China, 350202 \\ apwang@fjnu.edu.cn, b34168420@qq.com
}

Keywords: Zigbee, synchronization, DMTS, TPSN, Fusion Research.

\begin{abstract}
Time synchronization is important for Zigbee node. This paper takes advantage of the advantages of TPSN and DMTS synchronization algorithms. The integrated algorithm has lower complexity and lower energy consumption. It can be easily applied to a real wireless sensor network.
\end{abstract}

\section{Introduction}

Wireless sensor networks integrated sensor technology, Computer technology and communication technology. In the military, health, family, and business has a great many potential applications $^{[1]}$. High and low energy consumption is one of the important major signs about the measure of the performance of a wireless sensor network. We should let idle nodes as far as possible dormant, in order to save the the energy consumption of the node, of course, we can use TDMA in the MAC(Medium Access Control) layer, however, when we use this technology, it requires both nodes to achieve time synchronization.

NTP protocol is the Internet time synchronization protocol standard, because of the wireless sensor network has the volume, Power supply, storage capacity and other constraints. the wireless sensor network use wireless transmission and localized algorithm. So NTP protocol is not suitable for wireless sensor networks. GPS systems can also provide high-precision time synchronization, GPS antenna must be installed in open space and it needs high energy consumption, it is too expensive to attach on cheap node ${ }^{[2]}$.it is also not suitable for wireless sensor networks.

\section{THE DESIGN OF TIME SYNCHRONIZATION ALGORITHM}

\section{A. The analysis of DMTS algorithm}

DMTS(Delay Measurement Time Synchronization)algorithm ${ }^{[3]}$,it is based on the sender and receiver of the one-way time synchronization algorithm. The structure of this algorithm is simple and energy consumption is low. It was widely used. Figure 1 shows the algorithm of DMTS.

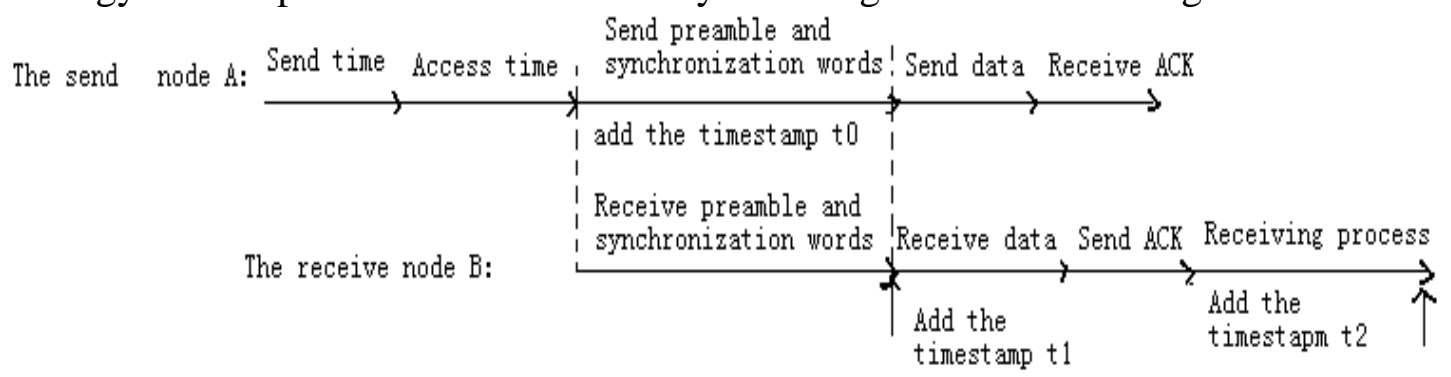

Figure 1 The theory of DMTS algorithm

\section{B. The analysis of TPSN algorithm}

TPSN(Timing-Sync Protocol for Sensor Networks)algorithm ${ }^{[4]}$, the network structure of TPSN is hierarchical. Based on the sender and the receiver of the bi-directional time synchronization algorithm, it can be divided into two stages, the first stage is to division-level stage, the second stage is a synchronous phase. The algorithm Figure 2 shows the algorithm of TPSN. 


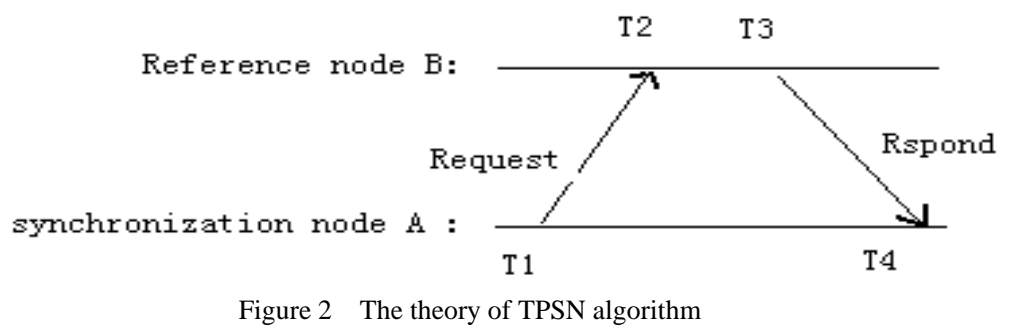

\section{The design of time synchronization algorithm based on DMTS and TPSN}

In this article, we make full use of the advantages of two algorithms. It can be divided into two paths, the main path and the secondary path. In the main path, it was made up from three router notes, we can see three router notes from the figure 3(A,B and E node).In the secondary path, it can divided into two Sub-networks. Sub-network 1 was made up of the router note B and two sensor nodes $C$ and D. Sub-network 2 was made up of the router note $E$ and two sensor nodes F and G. In the wireless sensor network, most of the node is composed of sensor nodes, they were used to collect data. Only a small number of nodes are composed of router notes, they were used to store, manage and send data. In the mainly path, the hops are more than the secondary path, in order to improve the accuracy of the network, we can use TPSN algorithm among router nodes. In the secondary path, we can use DMTS algorithm between the router node and the sensor node, it can not only reduce the network algorithm complexity, but also reduce the energy consumption of the entire network.

1. Time synchronization of the main path, first, node A by using the NLDE-DATA. request primitives, and set DstAddr parameter is 0xffff(Broadcast network address), node A use broadcast way to send time synchronization packet ${ }^{[5]}$. the packet contains the level of node $A$ and ID number of node $A$, the level of node $A$ is 0 , and set up the next hop is node $B$. After B node received the time synchronization packet from A node, note the level of B node is 1 , the ID number of parent node is A, and use TPSN algorithm to revise the offset time between two nodes. B node also use broadcast way to send time synchronization packet, and set up the next hop is E node. After C,D and E node receive the time synchronization packet from $B$, note the level is 2 , however, only $E$ node can synchronize with the A node.

2. Time synchronization of the secondary path, The main path of time synchronization is completed, then in the secondary path, we can use DMTS algorithm to Achieve time synchronization between nodes. if we not use high precision in the wireless network, we can choose a longer period time to Synchronous, in order to reduce energy consumption. In the sub-network 1,B node use broadcast way to send time synchronization packet, of course, this packet is different from the main path, and set up the next hop is $C$ and $D$ node, after $C$ and $D$ node receive the packet from the $\mathrm{B}$ node, modify their local clock to achieve synchronization with the node $\mathrm{B}$. The sub-network 2 use the same approach to achieve time synchronization.

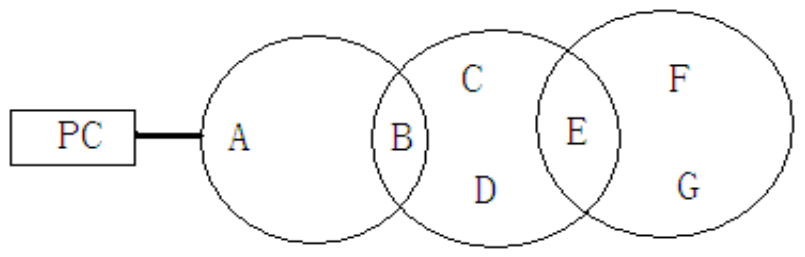

Figure 3 The experimental diagram of network nodes

\section{Program flow chart of the design algorithm}

The whole flow chart is shown in figure 4, the flow chart of main path is shown in figure 5,the flow chart of secondary path is shown in figure 6. 


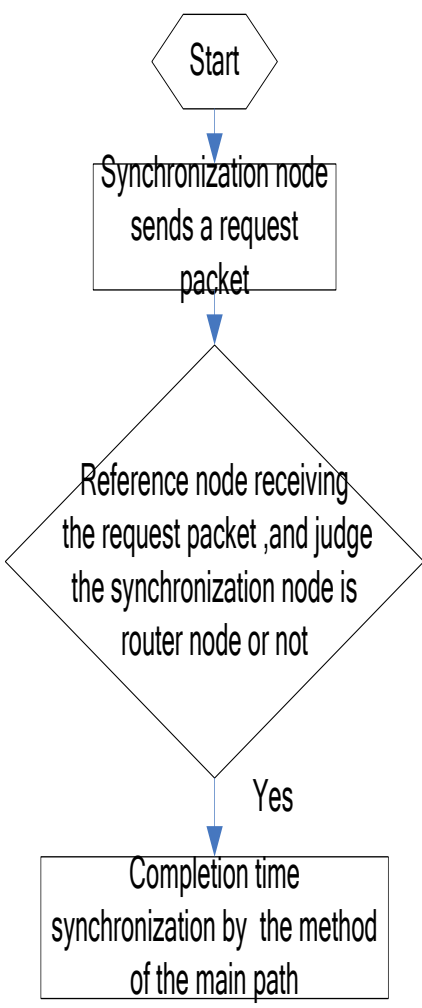

Figure 4 The whole flow chart

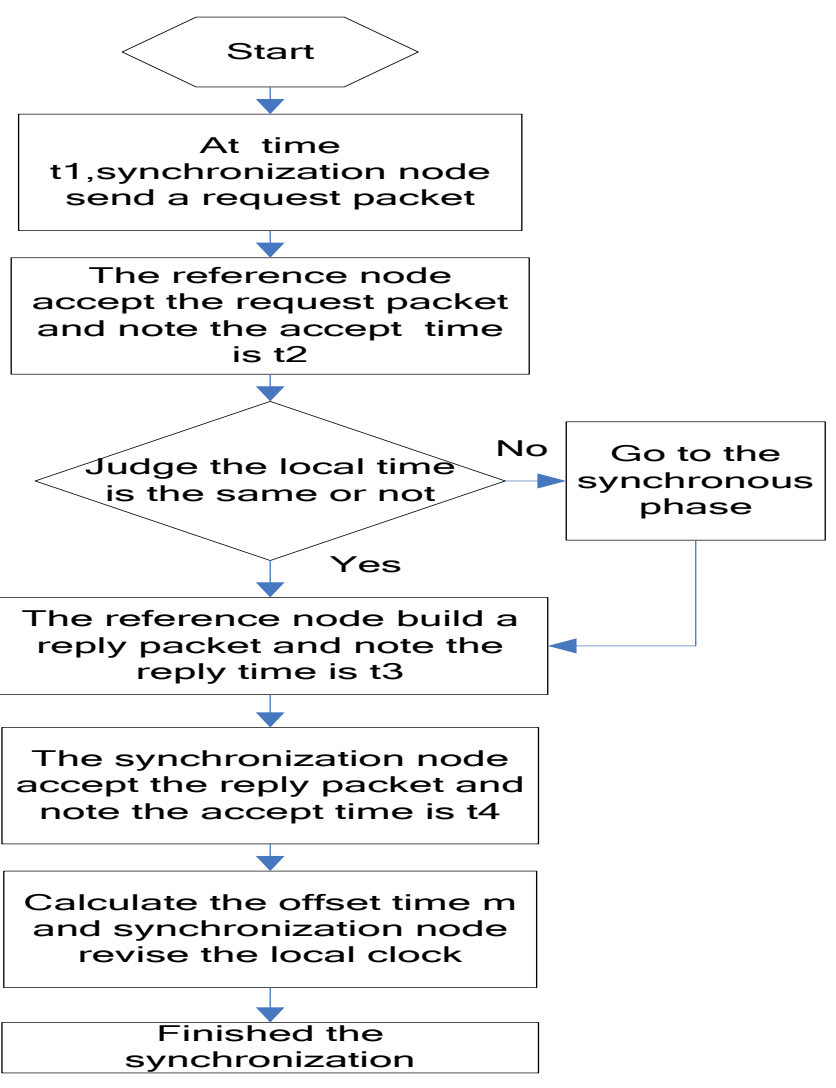

Figure 5 The flow chart of main path

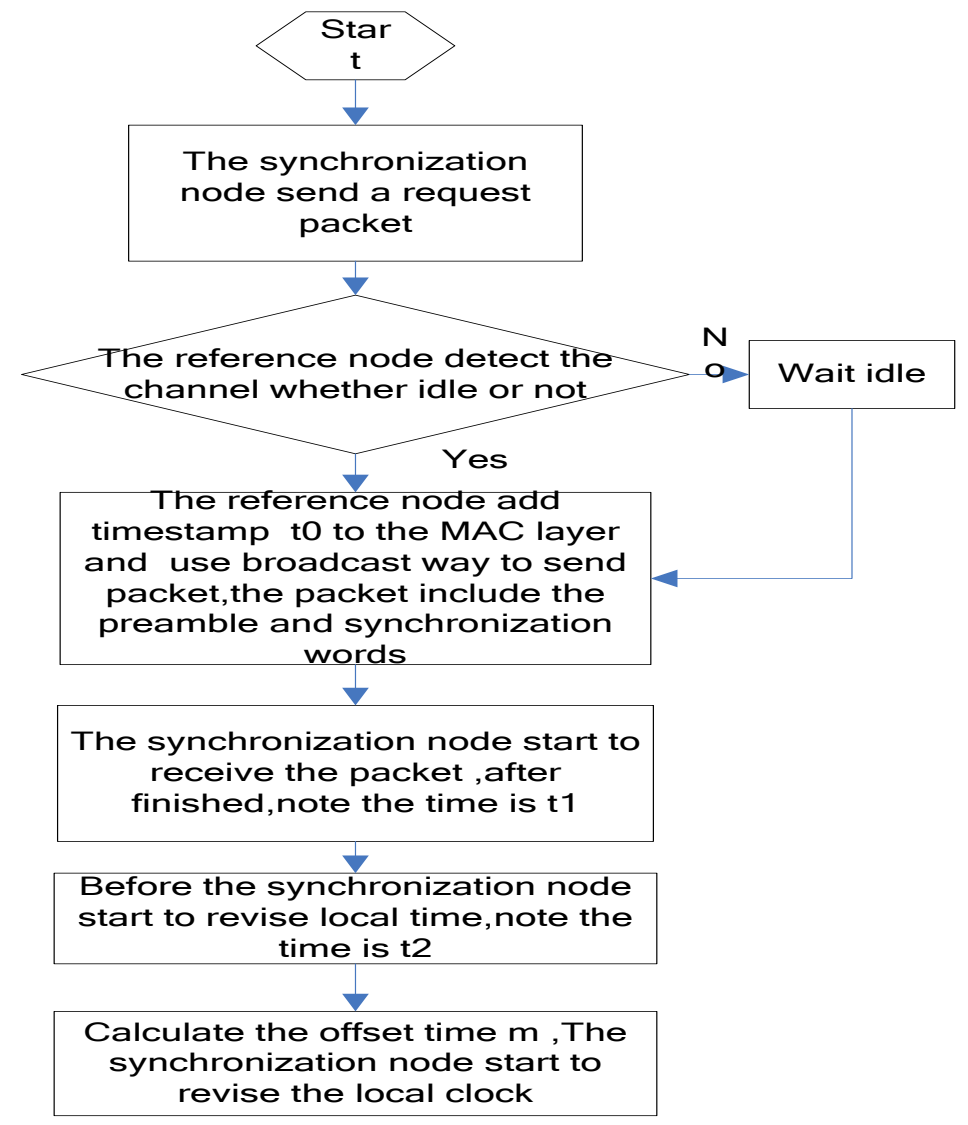

Figure 6 The flow chart of secondary path 


\section{CONCLUSION}

As we known,the precision of TPSN is higher than DMTS, but when we finished a time synchronization, The TPSN needs energy consumption of four messages, the DMTS only needs energy consumption of two messages, the innovation of this paper is combined two algorithm,we Full use of the advantages of both algorithm.The energy consumption and precision in between two algorithm.compared to the TPSN,the precision declined,but the precison is higher than DMTS and the energy consumption is lower than TPSN. Especially suitable for some Medium and small-scale wireless sensor networks which not require high precision, In wireless sensor network,not only keep the precision of the networks,but also reduce the energy consumption of the whole network.

\section{REFERENCES}

[1] Xianzhong Tian, Deng Chen and Tongsen Chen: Journal of Transduction Technology. China, vol. 21(2008), p. 1881.

[2] Zhetao Li, Renfa Li and Liangjiao Liu: Information Technology Journal. China, vol. 21(2009), p. 776.

[3] Ping Si: Intel Research Center, IR-TR-2003-64.2003.

[4] Hongxia Feng and Ying Zhou: Instrument Standardization \& Metrology.China, (2006) No. 6,P.30.

[5] Zhian Lv: Principles and Applications of ZigBee network (edtied by Beijing University of Aeronautics and Astronautics Press,China, 2008), P.120. 